\title{
Fingerlike Aggregates in Thin-Layer Electrodeposition
}

\author{
M.-Q. Lòpez-Salvans, ${ }^{1}$ P.P. Trigueros, ${ }^{1}$ S. Vallmitjana, ${ }^{2}$ J. Claret, ${ }^{1}$ and F. Sagués ${ }^{1}$ \\ ${ }^{1}$ Departament de Química Física, Universitat de Barcelona, Diagonal 647, Barcelona 08028, Spain \\ ${ }^{2}$ Departament de Física Aplicada i Electrònica, Universitat de Barcelona, Diagonal 647, Barcelona 08028, Spain
}

(Received 14 December 1995)

\begin{abstract}
Electrodeposition experiments conducted in a thin-layer horizontal cell containing a nonbinary aqueous electrolyte prepared with cupric sulfate and sodium sulfate gave rise to fingerlike deposits, a novel and unexpected growth mode in this context. Both the leading instability from which fingers emerge and some distinctive features of their steady evolution are interpreted in terms of a simple model based on the existing theory of fingering in fluids. [S0031-9007(96)00160-3]
\end{abstract}

PACS numbers: 81.15.Pq, 47.20.Ma, 81.10.Dn, 82.45.+z

After years of intense experimental and theoretical scrutiny, research on nonequilibrium growth patterns is presently facing the challenge of extracting universal features from the variety of phenomena investigated [1]. In this respect, finger morphology constitutes one of the "essential" growth forms which, however, has so far been circumscribed to the displacement [2] or spreading [3] of fluids. Here we report on our striking experimental discovery of fingering development in a completely unexpected scenario, that of thin-layer electrochemical deposition (ECD). Different from the most characteristic ECD morphologies, from the disordered fractals to the regularly patterned branching aggregates [4-16], the deposits obtained in these experiments show a small scale filament structure, which fills space densely, and is enclosed by a fingerlike, long-wavelength modulated envelope (Fig. 1). Although such a deposit texture largely prevents us from referring to a fully connected aggregate-solution interface and, consequently, invoking the concept of an equilibrium surface tension, it turns out that a strong similarity with the common fingering instabilities in fluids can be exploited once we assign a dynamically established effective surface tension to a boundary layer encompassing the growing deposit. The physicochemical properties of such a boundary layer, containing a hydrogen precipitate, are largely determined by the interplay between interfacial electrochemical effects and gravity-induced convective flows.

Most of the previous ECD experiments refer to purely binary electrolytes [4-13]. In contrast, experiments with electrolyte solutions containing added nondepositing cations, such as those reported here, are scarce in the literature [14-16]. Specifically, our thin-layer experimental cell, with thickness around $100 \mu \mathrm{m}$, is filled with a copper salt (typically $5 \times 10^{-2} M \mathrm{CuSO}_{4}$ ) containing a small amount of an alkaline salt with the same anion (normally $\mathrm{Na}_{2} \mathrm{SO}_{4}$ ) at concentrations comprised between $10^{-3} M$ and $10^{-2} M$. After some induction time and under constant and moderately high applied potentials, commonly between 10 and $30 \mathrm{~V}$, well-formed fingers such as those shown in Fig. 1, emerge and propagate steadily into the aqueous solution at velocities comprised between 10 and $30 \mu \mathrm{s}^{-1}$, depending on the applied potential. Both the fine texture and the dull reddish color of these deposits are markedly different from the bright red, tip-splitting structures obtained from analogous ECD experiments with pure $\mathrm{CuSO}_{4}$ solutions. The stage of steady finger propagation is normally interrupted by some morphological transitions to typically red, tip-splitting branching forms. This could be interpreted as a Hecker-like transition, well known in the standard ECD literature [16]. The apparently nonmetallic features of the fingerlike deposits were confirmed by scanning electron microscopy (SEM) and x-ray powder diffraction analysis. SEM observations indicate a highly agglomerated, structureless material. Much more enlightening are the x-ray data on the chemical composition: Up to $90 \%$ of the deposited material corresponds to cuprous oxide,

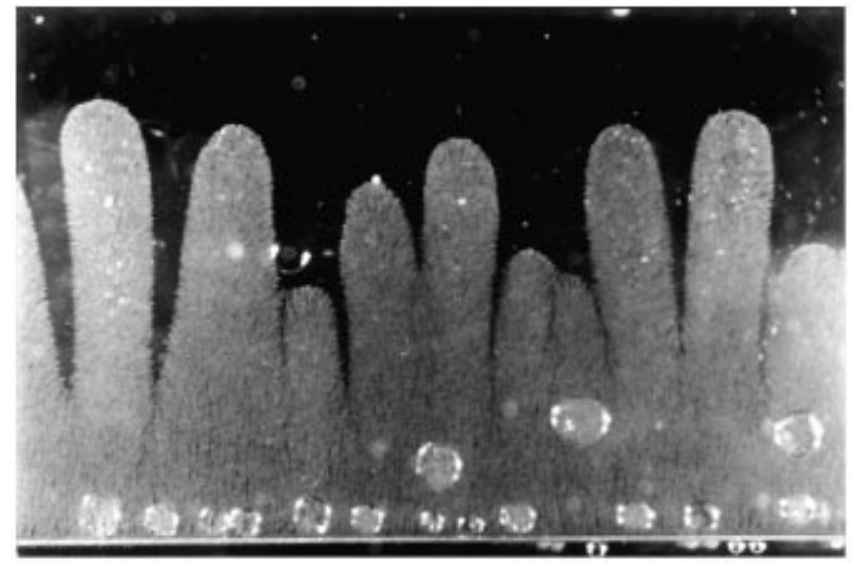

FIG. 1. Fingering pattern observed in a thin-layer ECD experiment with a nonbinary electrolyte. Growth conditions are $\mathrm{CuSO}_{4}\left(5 \times 10^{-2} M\right) / \mathrm{Na}_{2} \mathrm{SO}_{4}\left(2 \times 10^{-3} M\right)$; applied potential $\Delta V=25 \mathrm{~V}$; cell dimensions $(\mathrm{mm})$ are $110 \times 40 \times 0.1$. The area exposed corresponds to an electrode length of $16 \mathrm{~mm}$. Hydrogen bubbles are clearly observed in the picture. They originate in $\mathrm{H}_{2} \mathrm{O}$ reduction, triggered in turn, at high enough potentials, by the increased conductivity caused by $\mathrm{Na}_{2} \mathrm{SO}_{4}$ addition. 
as compared to a maximum of $30 \%$ for the usual copper aggregates.

Several additional experimental observations are worth mentioning in order to gain a better perspective of the observed phenomenon. Although the peculiar ECD morphology reported here is robust and fully reproducible, fingerlike patterns are only observed for an intermediate range of concentrations of the alkaline species and applied potentials [17]. In this last respect, exceedingly high electric fields lead to hydrogen accumulation, disturbing the appearance of any well-organized growth mode. In contrast, under excessively small driving electric forces, fingerlike patterns do not appear as the primary ECD morphology, but rather originate morphological transitions during growth, once higher enough current densities are attained. Such morphological transitions turn the typically red, tip-splitting copperlike deposits into the fingerlike ones described here. On the other hand, if we restrict our observations to the copper ECD experiments conducted so far, no signature of chemical specificity has been observed. Indeed, fingerlike patterns also appear when the composition of the electrolyte is changed either in its anionic species or in the nondepositing cationic ones. In this respect, experiments have been performed with acetate $\left(\mathrm{AcO}^{-}\right)$instead of sulfate $\left(\mathrm{SO}_{4}{ }^{2-}\right)$ and with lithium $\left(\mathrm{Li}^{+}\right)$, potassium $\left(\mathrm{K}^{+}\right)$, and cesium $\left(\mathrm{Cs}^{+}\right)$replacing the sodium cation $\left(\mathrm{Na}^{+}\right)$.

Following the destabilization of the initially dense and uniformly distributed deposit, screening effects, evidenced in Fig. 1, begin to affect finger growth. Immediately after this intermediate competing stage, surviving fingers grow independently and steadily. Thus, at this phase of pattern development, we attempted a direct comparison of this phenomenon with the standard viscous fingering problem. The classical result in this context is the celebrated Saffman-Taylor (ST) class of solutions describing the penetrating fluid profile as it invades a linear channel [18]. In Fig. 2 we compare a well-developed ECD finger with the ST solution corresponding to the vanishing surface tension limit. As shown in the figure the two contours are practically undistinguishable.

This rewarding agreement motivated us to pursue this classical analysis but referring this time to the very initial stage of the finger development. Generically, the onset of interfacial instabilities is characterized by a linear stability theory predicting their fundamental periodicities in terms of some appropriate control parameter. Translated into our context, this was checked by conducting a series of experiments varying the applied potential difference, and recording the number of incipient fingers, $N$, at different growth velocities, $v$. Summarized in Fig. 3, these results fit a power law, $N \sim v^{\beta}$ with $\beta \simeq 0.18$.

The last experimental observation worth mentioning concerns the role played by convection. Gravity-induced currents are well documented [19-21] in horizontal ECD cells due to the density contrast between the depleted

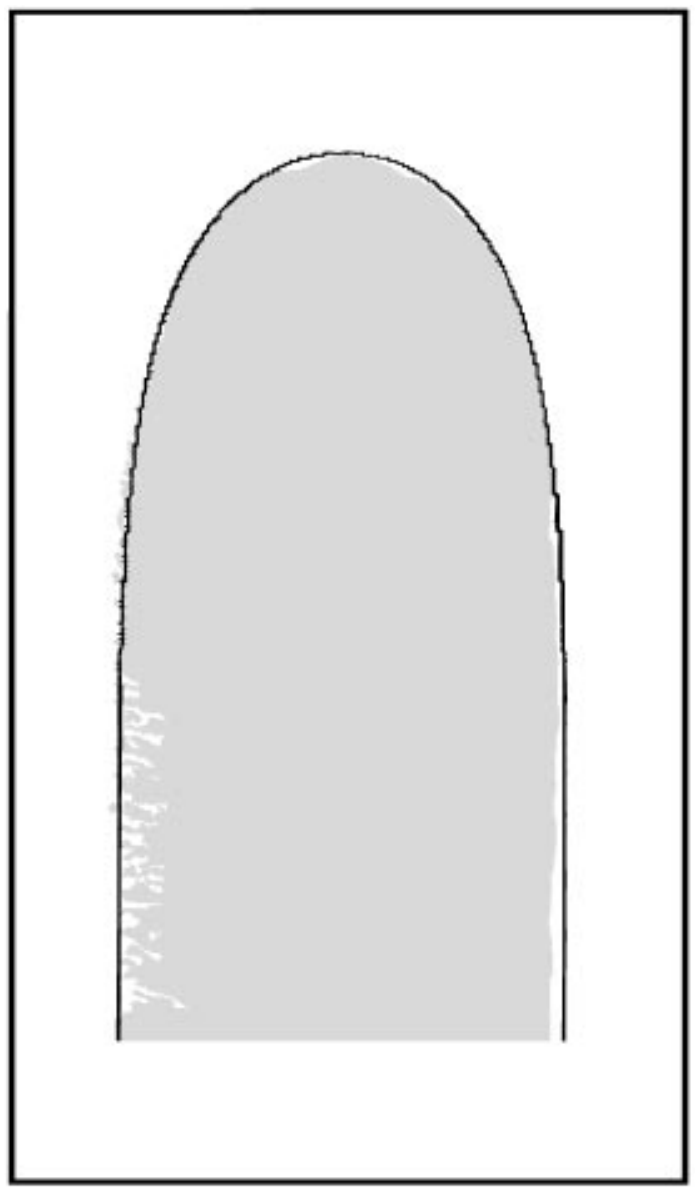

FIG. 2. The analytic Saffman-Taylor solution (so-called "Saffman-Taylor finger" or $\lambda=1 / 2$ particular solution for negligibly small capillary forces) is superposed to the envelope of a well-developed ECD finger. Growth conditions are $\mathrm{CuSO}_{4}\left(5 \times 10^{-2} M\right) / \mathrm{Na}_{2} \mathrm{SO}_{4}\left(6 \times 10^{-3} M\right) ; \Delta V=11 \mathrm{~V}$; finger width is ca. $4 \mathrm{~mm}$.

solution near the cathode and the bulk. Obviously, this effect can be largely suppressed by turning the ECD cell into a vertical configuration with the cathode facing downwards. Surprisingly enough, see Fig. 4, the deposits grown under this arrangement display their typical fine texture but are enclosed this time by an envelope which is free of any long wavelength modulation.

Let us now interpret these experimental observations. The presence of sodium sulfate increases the electric conductivity of the cell. As a result, higher density currents are attained than in growth conditions for purely binary electrolyte. This, in turn, triggers alternative chemical reactions concurrent with pure $\mathrm{Cu}^{2+}$ discharge. The most likely reaction is $\mathrm{H}_{2} \mathrm{O}$ reduction originating hydrogen evolution and the local $p \mathrm{H}$ increase. This would explain the prevalence of cuprous oxide in the deposit and suggests that the dominant electroaggregating process, concurrent with $\mathrm{Cu}^{2+}$ discharge, is the reduction of copper hydroxylated species $\mathrm{Cu}_{m}(\mathrm{OH})_{n}{ }^{(2 m-n)+}$. These poorly soluble intermediates [22] (hydrogel precipitate) accumulate within a 


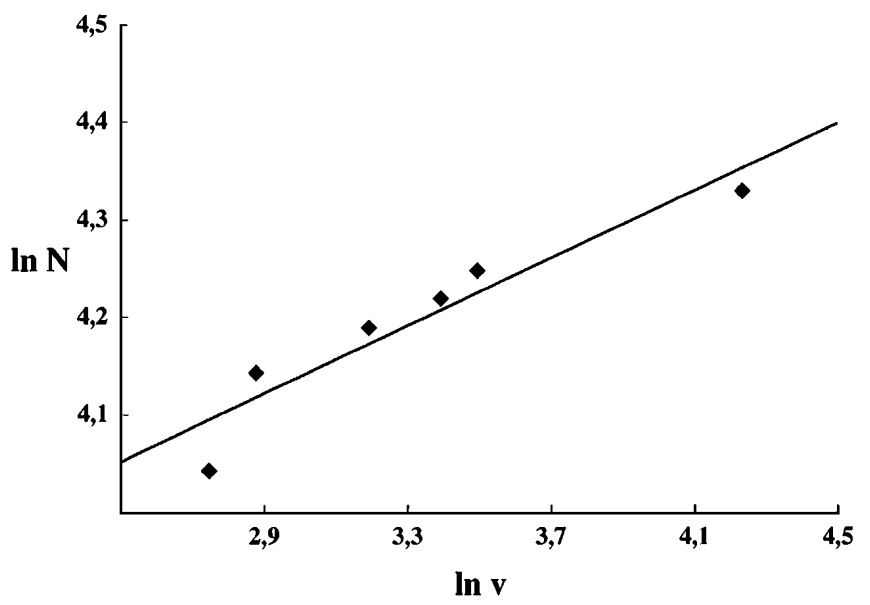

FIG. 3. Scaling of the number of incipient fingers with growth velocity. Growth conditions are $\mathrm{CuSO}_{4}\left(5 \times 10^{-2} M\right) / \mathrm{Na}_{2} \mathrm{SO}_{4}\left(3 \times 10^{-3} M\right)$; the applied potentials range from $15 \mathrm{~V}$ to $35 \mathrm{~V}$; cell thickness is $300 \mu \mathrm{m}$.

boundary layer, clearly shown in Fig. 5, separating the depleted solution, embedding the growing deposit, from the bulk. Indeed, the generic existence of much more tenuous concentration boundary layers than those encountered here is well reported in standard ECD experiments [19,23,24], the conclusion being that they are dynamically built up to a characteristic width $l_{c}$ by the growth process itself. Schlieren techniques applied to a series of complementary experiments conducted under usual ECD conditions, $0.2 \mathrm{M} \mathrm{CuSO}_{4}$ and cell thickness of $300 \mu \mathrm{m}$, led to a power law $l_{c}^{-\alpha} \propto v$ with $\alpha \simeq 1.5$. Since such optical techniques are not reliable when working with the more diluted solutions used in our fingering experiments, in what follows such a fit is taken for granted, this time applied to the width $l_{\mathrm{bl}}$ of the finger's boundary layer.

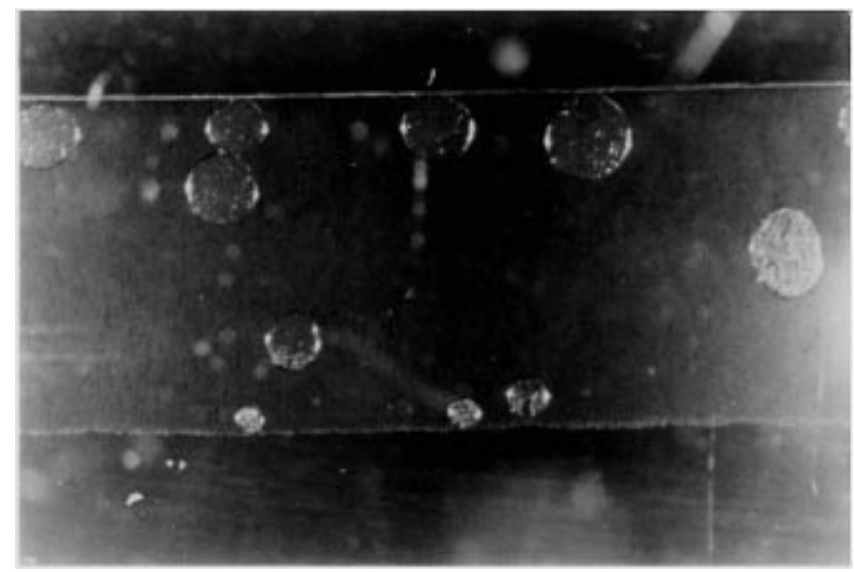

FIG. 4. Deposit obtained with our ECD cell in a vertical position with growth directed downwards. Growth conditions are $\mathrm{CuSO}_{4}\left(5 \times 10^{-2} M\right) / \mathrm{Na}_{2} \mathrm{SO}_{4}\left(2 \times 10^{-3} M\right) ; \Delta V=11 \mathrm{~V}$; cell thickness is $100 \mu \mathrm{m}$.
With these antecedents, let us adapt to our system the most genuine arguments concerning viscous fingering instabilities. In this respect, the rationale behind any fingering instability has to be sought in the opposite roles played by a destabilizing, typically Laplacian, mechanism and a restoring force, mediated by surface tension, which suppresses all but the smaller wave number disturbances. At the length scale at which our structure is formed, the first ingredient is associated with the electric driving force responsible for the $\mathrm{Cu}^{2+}$ migration and accretion of the depositing material. The second concept, however, should be replaced here by an effective dynamic surface tension $\sigma^{*}$, associated with the hydrogenlike boundary layer referred to above. Such surface tension modulates the displacement of the boundary layer, and that of the encompassed densely filling and tightly bound deposit, as it propagates into the bulk solution. In this respect, we indeed follow the arguments invoked in miscible fluid situations under moderately high concentration gradients $[25,26]$. Actually, such a diffusion-limited surface tension is predicted to be inversely proportional to the mixing layer thickness $[25,26]$. Then, it is a simple task to adapt the fundamental dispersion relation for a fingering instability $\left[k_{m} \propto\left(v \sigma^{-1}\right)^{1 / 2}\right]$ into a form directly suitable for comparison with our results in Fig. 3. Indeed, $N \propto$ $k_{m} \propto\left(v l_{\mathrm{bl}}\right)^{1 / 2} \propto v^{\beta}$, where $\beta=(\alpha-1) / 2 \alpha$, and taking $\alpha=1.5$ as determined previously, $\beta \simeq 0.16$. Given the simplicity of the model, the agreement with the experimentally determined scaling, $\beta \simeq 0.18$ is more than satisfactory. A final comment, fully coherent with the interpretation just proposed, would justify the observed finger suppression in convection-free situations. In this

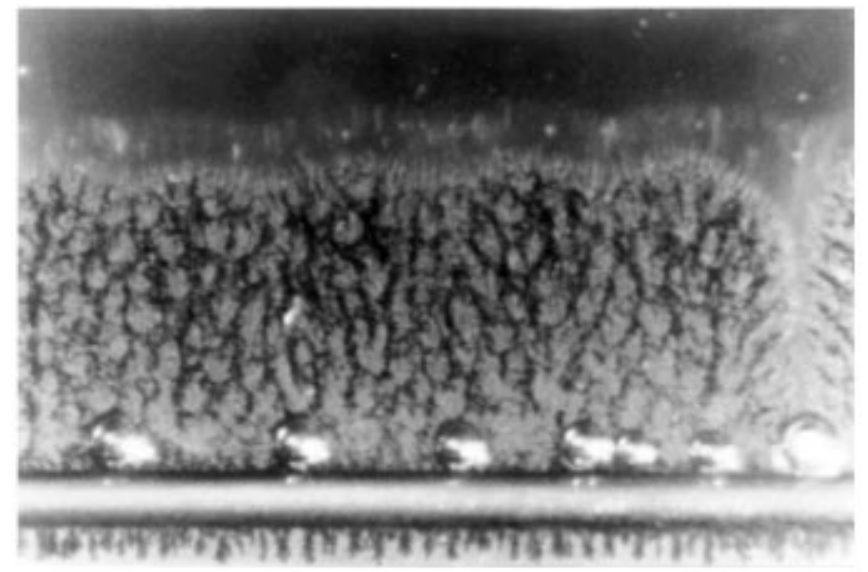

FIG. 5. An optical microscope image showing the boundary layer around the growing deposit where the intermediate $\mathrm{Cu}_{m}(\mathrm{OH})_{n}{ }^{(2 m-n)+}$ species accumulate. Mixing induced by natural convection (see text) stretches and dilutes this boundary layer, and it is easily imaged by the roll-like motions of its particulated precipitate. A thicker cell $(250 \mu \mathrm{m})$ is used here to facilitate observation. Growth conditions are $\mathrm{CuSO}_{4}\left(5 \times 10^{-2} M\right) / \mathrm{Na}_{2} \mathrm{SO}_{4}\left(7 \times 10^{-3} M\right) ; \Delta V=12 \mathrm{~V}$; image dimensions $(\mathrm{mm})$ are $4 \times 2.5$. 
case, the absence of effective fluid mixing and subsequent shrinkage of the boundary layer results in high enough values of the effective surface tension in order to prevent, in this way, the destabilization of the planar interface enclosing the growing deposit.

P.P. Trigueros and M.-Q. Lòpez-Salvans benefited from FPI grants from Ministerio de Educación y Ciencia (Spain). We also acknowledge the technical assistance of the Serveis Científico-Tècnics de la Universitat de Barcelona, and discussions with D. P. Barkey and J. Casademunt. This research was jointly supported by the Dirección General de Investigación Científica y Tecnológica (DGICYT), Spain, under Project No. PB93-0759, and Comissionat per Universitats i Recerca de la Generalitat de Catalunya.

Note added.-After the completion of this work, a reference appeared in the literature [J.R. De Bruyn, Phys. Rev. Lett. 74, 4843-4846 (1995)] commenting on fingeringlike, purely hydrodynamic instabilities in thinlayer ECD experiments. The essential difference with our paper is that there the instability refers strictly to electrochemical induced gravity currents, but the deposits themselves do not form fingerlike aggregates. Moreover, such instability is only observed when the anode is uphill of the cathode but never when the cell is leveled, as we use here to obtain fingerlike deposits.

[1] J.S. Langer, Science 243, 1150-1156 (1989); E. BenJacob and P. Garik, Nature (London) 343, 523-530 (1990); E. Ben-Jacob, Contemp. Phys. 34, 247-273 (1993).

[2] D. A. Kessler, J. Koplik, and H. Levine, Adv. Phys. 37, 255-339 (1988), and references therein.

[3] H. E. Huppert, Nature (London) 300, 427-429 (1982).

[4] M. Matsushita, M. Sano, Y. Hayakawa, H. Hondo, and Y. Sawada, Phys. Rev. Lett. 53, 286-289 (1984).

[5] Y. Sawada, A. Dougherty, and J.P. Gollub, Phys. Rev. Lett. 56, 1260-1263 (1986).

[6] D. Grier, E. Ben-Jacob, R. Clarke, and L. M. Sander, Phys. Rev. Lett. 56, 1264-1267 (1986).

[7] F. Argoul, A. Arneodo, G. Grasseau, and H. L. Swinney, Phys. Rev. Lett. 61, 2558-2561 (1988).

[8] P. Garik et al., Phys. Rev. Lett. 62, 2703-2706 (1989).

[9] J. R. Melrose, D. B. Hibbert, and R.C. Ball, Phys. Rev. Lett. 65, 3009-3012 (1988).
[10] V. Fleury, J.-N. Chazalviel, and M. Rosso, Phys. Rev. Lett. 68, 2492-2495 (1992); V. Fleury, M. Rosso, J.-N. Chazalviel, and B. Sapoval, Phys. Rev. A 44, 66936705 (1991); V. Fleury, J. H. Kaufman, and D. B. Hibbert, Nature (London) 367, 435-438 (1994).

[11] P. P. Trigueros, J. Claret, F. Mas, and F. Sagués, J. Electroanal. Chem. 312, 219-235 (1991); P.P. Trigueros, J. Claret, F. Mas, and F. Sagués, J. Electroanal. Chem. 328, 165-178 (1992).

[12] L. López-Tomàs, J. Claret, and F. Sagués, Phys. Rev. Lett. 71, 4373-4376 (1993).

[13] M. Wang, W. J.P. Van Enckevort, N.-B. Ming, and P. Bennema, Nature (London) 367, 438-441 (1994).

[14] R. M. Brady and R. C. Ball, Nature (London) 309, 225229 (1984).

[15] G. L. M. K. S. Kahanda and M. Tomkiewicz, J. Electrochem. Soc. 136, 1497-1502 (1989).

[16] A. Kuhn and F. Argoul, Phys. Rev. E 49, 4298-4305 (1994).

[17] P. P. Trigueros, J. Claret, and F. Sagués, Phys. Rev. E 49, 4328-4335 (1994).

[18] P. G. Saffman and G. I. Taylor, Proc. R. Soc. London A 245, 312-329 (1958).

[19] M. Rosso, J.-N. Chazalviel, V. Fleury, and E. Chassaign, Electrochim. Acta 39, 507-515 (1994).

[20] D. P. Barkey, D. Watt, Z. Liu, and S. Raber, J. Electrochem. Soc. 141, 1206-1212 (1994).

[21] J. M. Huth, H. L. Swinney, W. D. McCormick, A. Kuhn, and F. Argoul, Phys. Rev. E 51, 3444-3458 (1995).

[22] C.F. Baes, Jr., and R.E. Mesmer, The Hydrolysis of Cations (Wiley, New York, 1976).

[23] D. Barkey, J. Electrochem. Soc. 138, 2912-2917 (1991); D. Barkey, P. Garik, E. Ben-Jacob, B. Miller, and B. Orr, J. Electrochem. Soc. 139, 1044-1050 (1992).

[24] R. H. Cork, D. C. Pritchard, and W. Y. Tam, Phys. Rev. A 44, 6940-6943 (1991); E. Ben-Jacob, G. Deutscher, P. Garik, N.D. Goldenfeld, and Y. Lareah, Phys. Rev. Lett. 57, 1903-1906 (1986).

[25] D. D. Joseph, Eur. J. Mech. B Fluids 9, 565-596 (1990), and references therein; H.H. Hu and D. D. Joseph, Z. Angew. Math. Phys. 43, 626-644 (1992).

[26] According to Smith et al. [J. Colloid. Interface Sci. 80, 302 (1981)], the interfacial tension $s^{*}$ in a situation of miscible fluids is given as $s^{*}=\int_{-x_{0}}^{x_{0}}\left(\frac{\partial \phi}{\partial x}\right)^{2} d x$, where $x_{0}$ is a measure of the interfacial region and $\phi$ represents the local composition of the fluid phase. In normal situations, i.e., where these composition gradients are smoothed out by diffusion, $s^{*} \propto(\sqrt{D t})^{-1}$, indicating in this way that $s^{*}$ goes inversely proportional to the mixing layer thickness. 